\title{
Substitutional and orientational disorder in organic crystals: a symmetry-adapted ensemble model
}

Article

Accepted Version

Habgood, M., Grau-Crespo, R. and Price, S.L. (2011)

Substitutional and orientational disorder in organic crystals: a symmetry-adapted ensemble model. Physical Chemistry Chemical Physics, 13 (20). pp. 9590-9600. ISSN 1463-9076 doi: https://doi.org/10.1039/c1cp20249a Available at https://centaur.reading.ac.uk/36087/

It is advisable to refer to the publisher's version if you intend to cite from the work. See Guidance on citing.

To link to this article DOI: http://dx.doi.org/10.1039/c1cp20249a

Publisher: Royal Society of Chemistry

All outputs in CentAUR are protected by Intellectual Property Rights law, including copyright law. Copyright and IPR is retained by the creators or other copyright holders. Terms and conditions for use of this material are defined in the End User Agreement.

www.reading.ac.uk/centaur 
Central Archive at the University of Reading

Reading's research outputs online 


\title{
Substitutional and orientational disorder in organic crystals: a symmetry-adapted ensemble model
}

\author{
Matthew Habgood, ${ }^{* a}$ Ricardo Grau-Crespo $^{a}$ and Sarah L. Price ${ }^{a}$ \\ 5
}

Modelling of disorder in organic crystals is highly desirable since it would allow thermodynamic stabilities and other disorder-sensitive properties to be estimated for such systems. Two disordered organic molecular systems are modeled using a symmetry-adapted ensemble approach, in which the 10 disordered system is treated as an ensemble of the configurations of a supercell with respect to substitution of one disorder component for another. Computation time is kept manageable by performing calculations only on the symmetrically inequivalent configurations. Calculations are presented on a substitutionally disordered system, the dichloro/dibromobenzene solid solution, and on an orientationally disordered system, eniluracil, and the resultant free energies, disorder patterns, and system properties are

15 discussed. The results are found to be in agreement with experiment following manual removal of physically implausible configurations from ensemble averages, highlighting the dangers of a completely automated approach to organic crystal thermodynamics which ignores the barriers to equilibration once the crystal has been formed.

\section{Introduction}

20 Disorder is frequently found in organic crystals, with around $20 \%$ of all structures in the Cambridge Structural Database (CSD) ${ }^{1}$ reporting disorder information. It can take many forms. The crystal constituents may be in motion (dynamic disorder), ${ }^{2}$ or different constituents may occupy a continuum of different 25 positions (static but continuous disorder), or each constituent may occupy one of a limited number of possible states, or 'disorder components' (static, discrete disorder). ${ }^{3}$ Examples of this last kind include disorder in species substitution and orientational disorder in which the molecule is restricted to a few, well 30 defined, orientations. Static, discrete disorder has very rarely been studied in molecular crystals.

For crystallographers, a disordered system typically results ${ }^{3,4}$ in a diffraction pattern that refines to a crystal structure in which each site or sites is occupied by one of two (or more) possible 35 'components', with some approximately known probability - for example, the site is occupied by molecule A (component 1 ) or by molecule B (component 2) with a 50:50 probability. All that can be inferred is that within some large volume of the crystal, half of the total sites are occupied by A and half by B. Any probability is

40 possible in principle, for example 74:26 found in a crystal of eniluracil. ${ }^{5}$ Any pattern in the distribution of A and B (on a superunit-cell scale), is exceptionally difficult to elucidate. The characterization of the crystal is therefore incomplete. A method for inferring short-range ordering in disordered systems based on 45 diffuse scattering is maturing, but requires high-quality data and elaborate analyses. ${ }^{6-9}$ Superspace analysis ${ }^{10}$ can be used to elucidate whether the 'disordered' sites possess incommensurate periodicity, but this phenomenon is relatively rare in disordered solids.

50 The resultant uncertainties affect computational modellers attempting to estimate the stability or properties of a disordered system. A simple approximation is to perform calculations for only the 'pure' solids, containing just one component, and interpolate the results to the mixed solid in a straightforward way. ${ }_{55}$ An example would be calculating the lattice energy of each endmember of a 50:50 disordered system, taking the average, and then adding a configurational entropy term for the mixing of the two (equal to $R T \ln 2$, in this case). While this approach may provide a working value, it is clearly unsatisfactory, as the mixing 60 entropy term assumes that the components are perfectly randomly distributed and ignores the differential interactions between them. Furthermore, it gives no insight into the true nature of the disorder. 
<smiles>[X]c1ccccc1[X]</smiles>

(a)

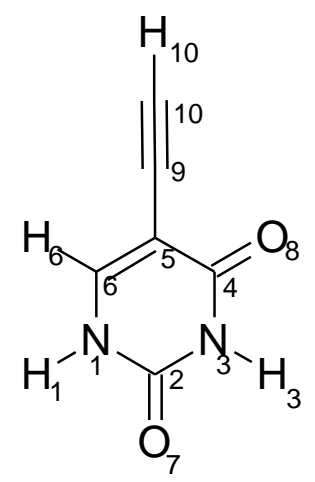

(b)
Fig. 1 The two systems (a) a $p$-dihalobenzene; $\mathrm{X}=\mathrm{Cl}, \mathrm{Br}$. The two molecules are the disorder components of the solid solution, (b) 5-ethynyl 5 uracil (eniluracil). The disorder components are the orientations produced

by a $180^{\circ}$ rotation around the $\mathrm{C}_{10}-\mathrm{C}_{9}$ axis relative to the crystal axes.

Disorder is also a challenge in predicting the crystal structures of organic molecules from the molecular diagram and quantitative models of intermolecular forces. ${ }^{11-15}$ Crystal structure prediction

10 (CSP) methods produce a set of hypothetical crystal structures, or 'crystal energy landscape', which sometimes include multiple, similar crystal structures of roughly the same energy. In these cases there could be a disordered crystal structure, which is stabilised by configurational entropy. This kind of landscape has 15 been found to correspond to actual disorder in eniluracil, ${ }^{5}$ chlorouracil, ${ }^{16}$ cyclopentane ${ }^{17}$ and caffeine. ${ }^{15}$

There have been several studies on the modelling of discrete disorder at levels other than the infinite dilution limit in ionic and metallic systems using a combination of configurational 20 ensemble statistics with classical or quantum-mechanical models for obtaining the energies and relaxed structures of configurations. The ensemble approach incorporates temperature effects via conventional statistical mechanics, thus allowing the modelling of intermediate situations between the perfect order 25 and the perfect disorder cases. Two main approaches to generating an ensemble have emerged. One is the enumerationbased 'symmetry-adapted ensemble' approach. All the possible configurations of the disorder components within a given supercell are identified and their energies evaluated. The 30 computation time is made tractable by consideration of the symmetry of the supercell and performing calculations only on the symmetrically inequivalent configurations. This is implemented in the SOD ('Site-Occupancy Disorder') program, ${ }^{18}$ which has been used for modelling of many inorganic systems. ${ }^{19-}$

${ }_{35}{ }^{23}$ It has also been implemented in recent versions of the periodic electronic structure program CRYSTAL. ${ }^{24} \mathrm{~A}$ variation on the symmetry-adapted ensemble approach has used counting of nearest neighbours to reduce the size of the unique configuration space. $^{25}$ The other approach is 'Monte Carlo with exchange' $40(\mathrm{MCX})^{26}$ which involves the use of Monte Carlo (MC) simulations with a modified moveset that includes the exchange of one disorder component for another at a randomly selected site. Various modifications of the Monte Carlo method have been used, ${ }^{27-32}$ including parallel tempering. ${ }^{33}$ Both major approaches 45 build on slightly older schemes that attempt full enumeration without symmetry adaption, ${ }^{34}$ or random selection of configurations. ${ }^{35}$ Applications of these methods have ranged from alloys to defective metal oxides, and includes the only previous study of 50:50 disorder in a molecular crystal. ${ }^{13}$

50 A third approach is the use of "special quasi-random structures", where components are distributed within a supercell in a way that mimics the short-range disorder of a random structure ${ }^{36}$. These can be useful, but the approach is unable to describe varying levels of disorder (e.g with temperature) for a given system.

${ }_{55}$ Of the two main approaches, MCX is the faster in generating a disorder model and hence allows for a larger supercell, while the symmetry-adapted ensemble approach guarantees a complete set of configurations for a given supercell. However, it is not yet clear which of the two has the advantage in terms of accuracy, 60 and it seems likely that the approaches are complementary, with different methods being preferable for different materials.

Neither approach has been applied to molecular crystals. Instead, MC algorithms incorporating changes in unit cell size and shape have been used to probe dynamic, orientationally disordered 65 phases ('plastic phases') and the static distributions that can be obtained by cooling them. ${ }^{37-39}$ These techniques are entirely suitable for the dynamically disordered systems or static systems with low barriers to rearrangement. Higher barriers to switching between components will prevent these approaches from 70 exploring the configuration space, so they cannot be used for studying static, binary systems, excluding a whole class of molecular crystals from consideration. The application of one of the major approaches used for inorganic crystals to static disorder would therefore allow the study of a new class of organic, 75 molecular systems.

While the basic approaches are transferable, molecular crystals are different in a number of ways to atomistic systems. Most importantly, molecular crystals are frequently orientationally disordered, in addition to static disorder that may arise from 80 molecular substitutions and vacancy-formation. Secondly, energy calculations in molecular systems require different methods to ionic or metallic systems, because of the importance of dispersion forces and intermolecular electrostatic interactions.

There are several examples of organic crystals that display 85 substitutional disorder, ${ }^{40-42}$ including solid solutions of carbamazepine and dihydrocarbamazepine ${ }^{43}$ and of chloro and methyl $o$-benzoic acids. ${ }^{44}$ Many more have been shown to display orientational disorder, $6,7,17,45-48$ including the pesticide chlorothalonil $^{49}$ and stimulant caffeine. ${ }^{50-52}$ Hence disorder 90 modelling is potentially valuable for understanding the solid forms developed by the pharmaceutical industry.

In this study, the symmetry-adapted ensemble approach (a choice discussed in Section 4.1) is applied to disorder in organic, molecular crystal systems. The core SOD functionality is used for 95 the generation of symmetry-unique supercell configurations, while the program DMACRYS ${ }^{53}$ is used for calculation of the energies of individual configurations using realistic, anisotropic intermolecular potentials. To exemplify the combined SOD/DMACRYS approach, calculations are presented on two 100 systems (see Fig. 1), representing respectively substitutional and orientational disorder. The first system is the solid solution of $p$ dichlorobenzene $(\mathrm{pDCB})$ and $p$-dibromobenzene $(\mathrm{pDBB})$. The two compounds are known to have isomorphic polymorphs (Cambridge Structural Database refcodes DCLBEN07 ( $\alpha$ - 
polymorph), CISTON) and the solid solution, which is isomorphic to both, has been studied experimentally ${ }^{54,55}$ and in the only previous molecular crystal binary static disorder study, ${ }^{13}$ although none of the results have been quantitatively conclusive. 5 The second system is 5-ethynyluracil (eniluracil). This compound has already been the subject of a thorough experimental and CSP study. ${ }^{5}$ Single crystal X-ray diffraction studies of four crystals showed variable disorder, after the crystal energy landscape suggested this would rationalise the variable properties of 10 microcrystalline samples. A disorder study of eniluracil will also be qualitatively applicable to the isomorphic systems
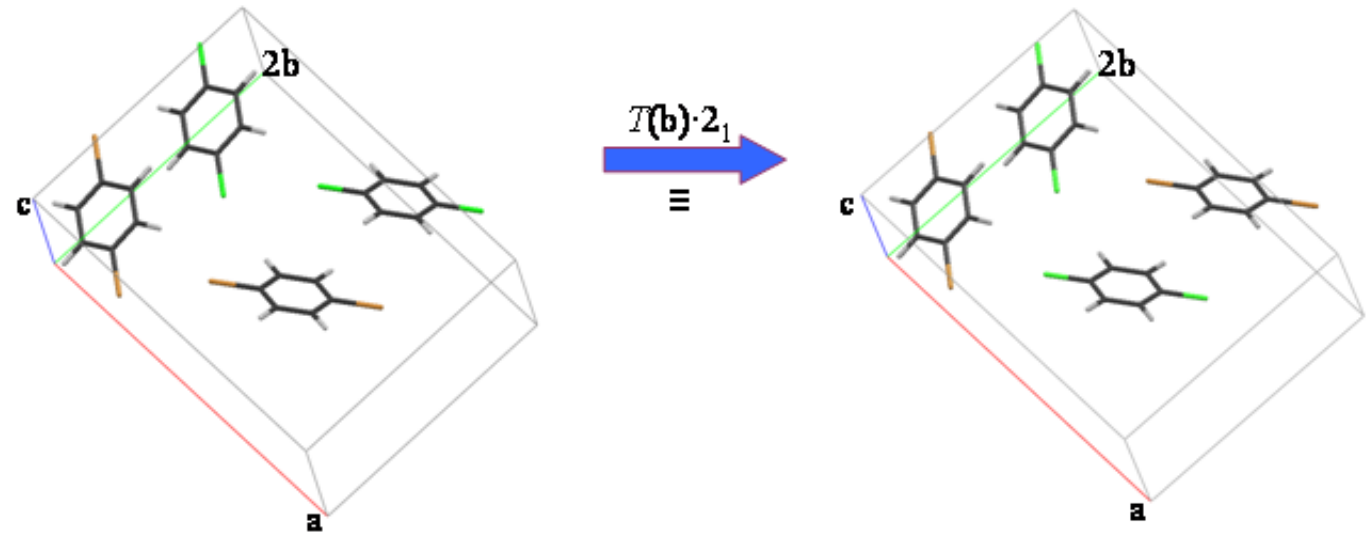

Fig. 2 Two symmetrically equivalent configurations in a $1 \times 2 \times 1$ supercell of the $P 2_{1}$ solid solution of $\mathrm{pDCB}$ and pDBB. The configuration of two pDBB substitutions on the left is transformed into that on the right by the product of a $2_{1}$ screw axis and a translation in the $\mathbf{b}$ lattice vector denoted by $T(\mathbf{b}) \cdot 2$.

5-chloro and 5-bromouracil. ${ }^{16}$ It is therefore an ideal candidate 20 for validation of the method on an orientationally disordered system, where the two disorder components are related by rotation about the ethynyl group.

For both of these systems, free energies are calculated both for observed and hypothetical disorder ratios, and the patterns of ${ }_{25}$ disorder predicted within different structural motifs are identified.

\section{General Method}

\subsection{Summary of the 'symmetry-adapted ensemble' technique $^{18}$}

30 The symmetry adapted ensemble technique assumes a crystal structure in which at least one site has been identified as disordered. As the first step, a supercell of the crystal lattice must be chosen. The larger the supercell, the more accurate the results will be. The number of sites which could be disordered within the

35 chosen supercell is denoted $N$. A disordered system is modelled as an ensemble of copies of the supercell, in which differing combinations of the disorder components are placed at the disordered site(s). The different supercell structures are called 'configurations'. For a symmetry-adapted ensemble, all possible 40 configurations of the chosen supercell are taken into account. However, the symmetry of the crystal lattice means that only a subset of these will actually be distinct from each other. The process of identifying the symmetry-unique configurations is straightforward for binary disorder, and is automated by the ${ }_{45}$ program SOD. For $N$ disordered sites, there are a total of $2^{N}$ configurations (including varying compositions for substitutional disorder). These are considered sequentially. For each in turn, all the configurations that are symmetrically equivalent are identified by application of each of the symmetry operators of the supercell 50 (consisting of the space group symmetry operators combined with each of the lattice translation vectors within the supercell). Configurations that are marked as equivalent to one already generated are counted but not considered further (see Fig. 2). Once all possible configurations have been generated, either in 55 sequence or by symmetry equivalence, a list is obtained of (i) all symmetry-unique configurations and (ii) their 'degeneracies' (denoted $\Omega_{m}$ for configuration $m$ ), in terms of symmetry equivalent configurations. For computational convenience, this process is usually broken up into separate steps for a number $n=$ ${ }_{60} 0,1,2, \ldots, N$ of disordered sites at which substitutions are allowed to occur.

In group theoretical terms, this process can be thought of as enumeration of the invariant subspaces of a basis of $n$ site substitutions (which, in itself, is a direct product of $n$ spaces of ${ }_{65}$ substitution at a single disordered lattice site). This is achieved by direct calculation of the matrix representation of each of the symmetry elements in the expanded group given by the product of the lattice translations within the supercell with the space group symmetry elements. Higher order space groups (i.e. a 70 higher symmetry system) will yield fewer symmetrically inequivalent configurations. The $\mathrm{pDCB} / \mathrm{pDBB}$ solid solution and eniluracil have space groups with $Z=2$ and $Z=4$ (i.e. $P 2_{1}$ and $P 2_{1} / c$ respectively). The number of symmetry operations meant that computational constraints limited the supercells used to 16 75 molecules, giving $65536\left(=2^{16}\right)$ configurations. This will typically reduce to $\sim 1000-5000$ symmetry independent configurations under the symmetry operators of an organic crystal. This is a relatively small number of molecules in the simulation by the standards of Monte Carlo calculations. However, in both systems, 
16 molecules allow all nearest neighbour interactions to be captured, and these will be the most important in determining the stability of the disordered system.

Once the symmetry-unique configurations are identified, crystal 5 structures can be written out and energies (and other properties) calculated using separate software. Average stabilities and properties for the whole ensemble can then be evaluated (see Section 2.3). For this study the basic SOD program was augmented with routines to automatically generate symmetry 10 operators for a supercell from the symmetry information contained in SHELX (.res)-format crystal structure files.

For each symmetry-unique configuration with lattice energy $E_{m}$ relative to infinitely separated molecules, the degeneracy $\Omega_{m}$ gives a 'reduced energy'

${ }_{15} \tilde{E}_{m}=E_{m}-T S_{m}$

where

$$
S_{m}=R \ln \Omega_{m}
$$

The reduced energy is a free energy for the configuration $m$ that takes into account only the entropy arising from its degeneracy. 20 All energies and entropies reported here are given per molecule, as typically done in organic crystal thermodynamics. A temperature of $T=298 \mathrm{~K}$ is used throughout this study.

\subsection{Energy and properties calculations using DMACRYS}

${ }_{25}$ In this study, individual supercell configurations were relaxed using a realistic model of the intermolecular potential, and their final energies calculated, using the program DMACRYS ${ }^{53}$ The electrostatic part of the intermolecular potential was calculated from a representation of the isolated molecule electron density in 30 the form of multipoles distributed to atomic sites. This allows local anisotropy in the charge distribution to be accurately modelled. Multipoles up to rank 4 were used with the electrostatic energy expansion truncated at $R^{-5}$. The multipoles were calculated using the GDMA2.2 program $^{56}$ using isolated 35 molecule wavefunctions produced using the program GAUSSIAN03 $^{57}$ at the MP2 level of theory with a $6-31 \mathrm{G}(\mathrm{d}, \mathrm{p})$ basis set. (The molecular geometry was optimized at the same level of theory.) All other terms in the intermolecular potential were represented with an exp-6 potential that had been fitted to 40 organic crystal structures $;^{58-61}$ in the case of bromine, the parameters used were derived from a previously published ${ }^{62}$ anisotropic potential with the anisotropy removed. The anisotropy makes a negligible difference in the structures examined here. All input files required by DMACRYS to model each supercell 45 configuration were assembled automatically using simple augmentations to the SOD code. As is standard for organic crystals, all lattice energies are calculated and quoted relative to infinitely separated molecules.

Mechanical properties were calculated separately for individual 50 configurations using the properties module ${ }^{63}$ built into DMACRYS, which calculates approximate elastic constants based on the rigid-body second derivatives of the intermolecular energy. These properties were then averaged over an ensemble.

\subsection{Calculating ensemble averages}

${ }_{55}$ It is desirable to study energy as a function of the overall disorder ratio (denoted $\tau$ ), and to use the whole set of configurations to do so. However, there is a complication. The set of configurations includes a range of discrete ratios, $n / N$ (where $n=0,1,2, \ldots, N$ ). Hence, any given specified ratio (e.g. an experimentally derived 60 ratio of 73:27) is unlikely to correspond exactly to any of these, due to the limitations of the finite supercell.

Disorder is a problem of variable composition, and it is therefore appropriate to employ the grand canonical ensemble, ${ }^{64}$ and hence the chemical potential, $\mu$. In this study, $\mu$ is employed in a general ${ }_{65}$ sense as an external constraint that will yield the desired disorder ratio $\tau$. This constraint reflects a real redistribution in the probability of finding the different configurations (relative to $\mu=$ 0 ) and arises from various physical origins discussed in section 4.1.

70 The chemical potential yields an additional weighting $\mu n$ for configurations with $n$ substitutions of disorder component 2 for component 1 , for the purposes of ensemble averages. The probability of finding each symmetry-unique configuration within the ensemble is then given by

${ }_{75} P_{m}=\frac{1}{\Xi} \exp \left(\frac{-\tilde{E}_{m}+\mu n_{m}}{R T}\right)$,

where $n_{m}$ is the number of substitutions in configuration $m$ and $\Xi$ is the grand canonical partition function,

$$
\Xi=\sum_{m} \exp \left(\frac{-\tilde{E}_{m}+\mu n_{m}}{R T}\right)
$$

${ }_{80}$ To determine energies and properties for a system with a specified disorder ratio, $\tau$, the chemical potential, $\mu_{\tau}$, that will yield this ratio is determined from the equation

$$
\tau=\sum_{m} P_{m}\left(\frac{n_{m}}{N}\right),
$$

by a method described in the Supporting Information (Section $\left.{ }_{85} \mathrm{~S} 1\right)$.

System properties are obtained from the values calculated for the individual configurations using the probabilities in eqn (3). The free energy is calculated using the standard formula

$$
A=-R T \ln \Xi+\mu_{\tau} \tau
$$

90 and is considered per molecule. Pressure is approximated to zero throughout, as is standard practice for organic crystal systems at room temperature and pressure, ${ }^{65}$ so the Gibbs and Helmholtz free energies are identical. 


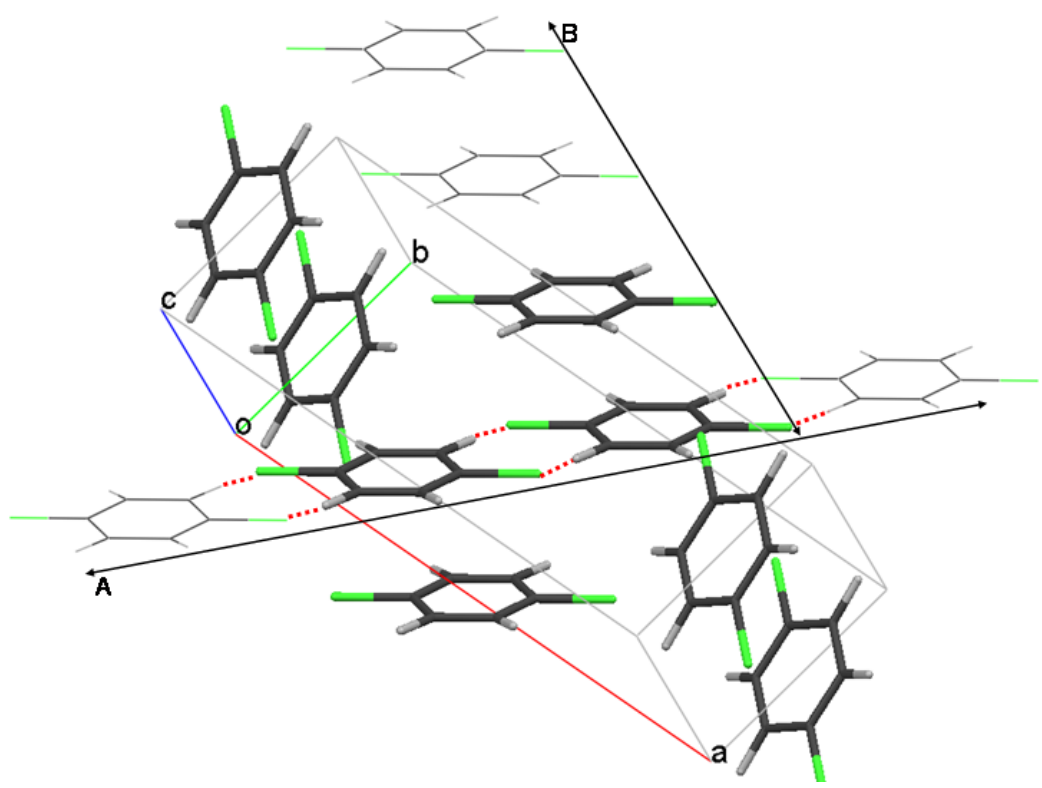

Fig. 3 The crystal structure of $\alpha$-pDCB. All molecules outside the conventional unit cell are shown in wireframe. The main structural features are the ribbons joined by $\mathrm{H} \cdots \mathrm{Cl}$ close contacts (one shown, A, horizontal, close contacts marked by broken lines) and the molecular stacks (one shown, B, vertical). The crystal structure obtained from the CSD for $\alpha$-pDCB is $P 2_{1} / a$ with $Z^{\prime}=0.5$. Since internal molecular symmetry does not affect moleculelevel disorder, this was transformed to $P 2_{1}$ with $Z^{\prime}=1$.

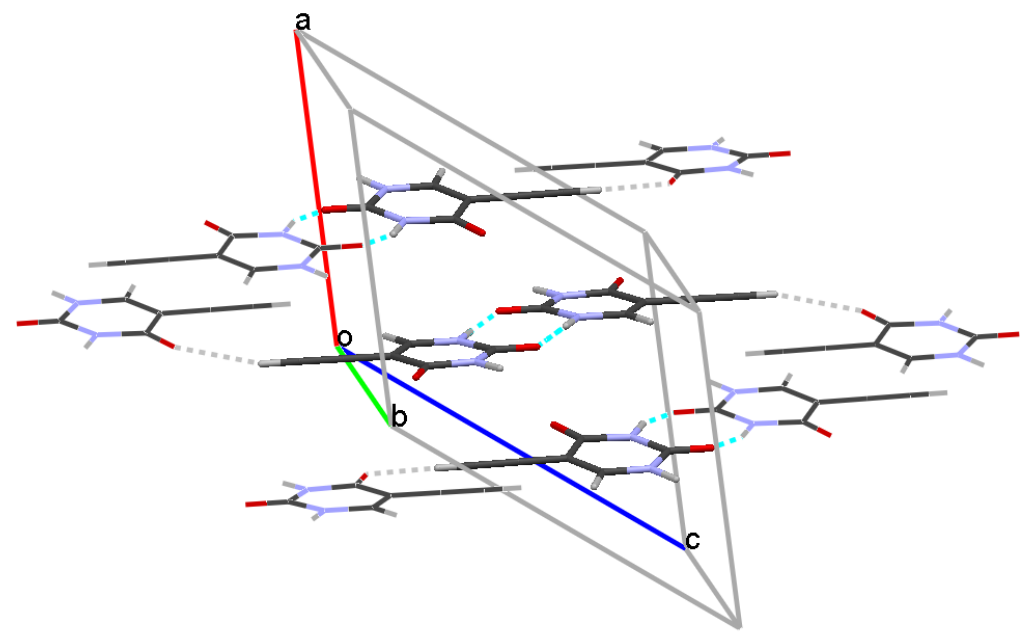

Fig. 4 The major component of the crystal structure of eniluracil (as modeled by computed structure $a k 56^{5}$ ). All molecules outside the unit cell are shown in wireframe. Stacking is parallel to the $a$ axis. Hydrogen bonds and close contacts are marked by broken lines. 


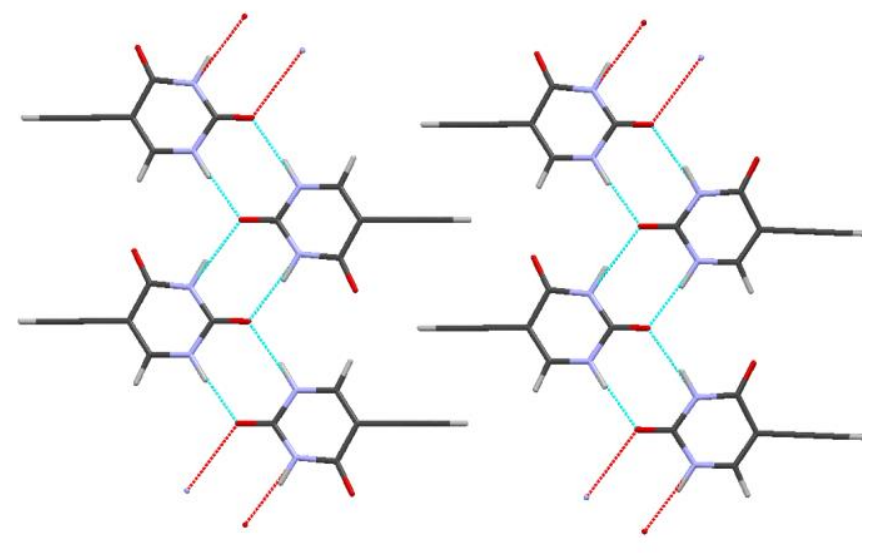

(a) (b)

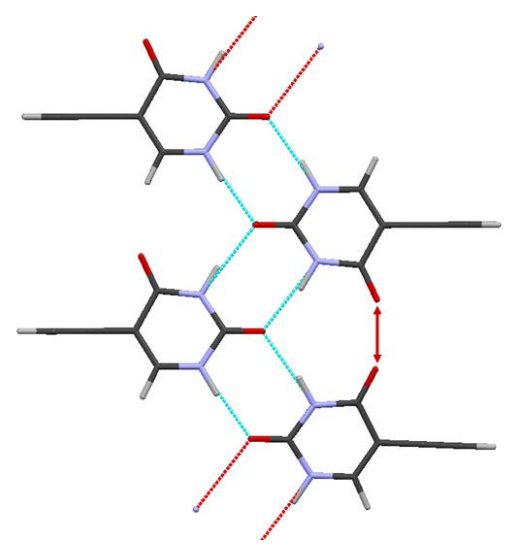

(c)

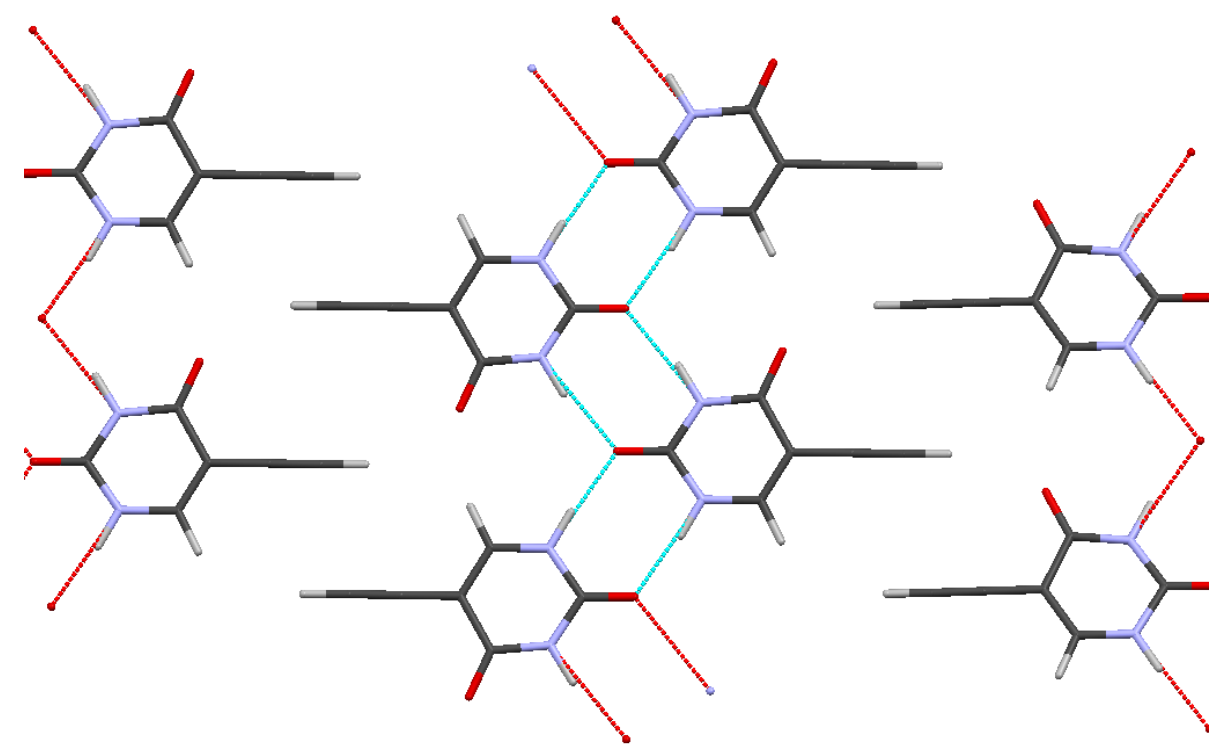

5 Fig. 5 Hydrogen-bonded base-paired ribbons in the eniluracil structure; (a) a uniform non-polar ribbon. All molecules are in the same disorder component; by crystal symmetry, the molecules on opposite sides of the ribbon are therefore in opposite orientations, (b) a polar ribbon (the two 'sides' of the ribbon are in opposite disorder components, and therefore the same orientation), (c) a ribbon that is neither polar nor non-polar, with a close $\mathrm{O} \cdots \mathrm{O}$ contact (marked by an arrow), (d) ribbons in a sheet with the interdigitating molecules matching in orientation (right hand side) and anti-parallel (left hand side).

Note that all free energies mentioned in this section include only 10 the entropic contribution from configurational disorder, and are therefore not the complete free energy for the system. Entropy arising from the thermal motion of atoms and molecules was neglected. However, if thermal motion entropies can be obtained, they may be added into the reduced energy for each 15 configuration.

For each system, structural features such as hydrogen bonding motifs and stacking affected by the disorder were evaluated for each configuration. Average frequencies for the occurrence of these features were calculated as grand canonical ensemble 20 averages, using the probablities in eqn (3) to quantify the content of the disordered system in terms of the hydrogen bonding and packing motifs.

\subsection{Calculations on $p$-dichlorobenzene/p-dibromobenzene 25 solid solution}

Structures containing pDBB were generated by pasting a gasphase optimized molecule of pDBB into a supercell of the crystal lattice of DCLBEN07 (see Fig. 3). The pure DBB crystal obtained in this way (i.e. by substituting all molecules for $\mathrm{pDBB}$ ) 30 was a very close match to the experimental pDBB structure. ${ }^{66}$

Relaxation using the intermolecular potential discussed in section 2.2 was used to take account of the effects of the pDBB molecules. A $2 \times 2 \times 2$ unit cell (16 molecules) was used, giving 
4216 symmetry-unique configurations. This supercell captures all nearest neighbour interactions, and is therefore sufficiently lareg for the current study. The stability of the solid solution was assessed using free energies of mixing, $A_{m i x}=A\left(\mathrm{pDCB}_{1-\tau} \mathrm{pDBB}_{\tau}\right)$ ${ }_{5}(1-\tau) A(\mathrm{pDCB})-\tau A(\mathrm{pDBB})$, where the free energy of the mixed system was calculated using eqn (6), and the free energies of the pure components were approximated by their lattice energies of $68.82 \mathrm{~kJ} \mathrm{~mol}^{-1}$ and $-61.21 \mathrm{~kJ} \mathrm{~mol}^{-1}$, for $\alpha$-pDCB and pDBB respectively. Potential energies of mixing $\left(E_{\text {mix }}\right)$ were calculated 10 analogously, with

$E\left(\mathrm{pDCB}_{1-\tau} \mathrm{pDBB}_{\tau}\right)=\Sigma P_{\mathrm{m}} E_{\mathrm{m}}$, and the entropic energy $\mathrm{T} S_{\text {mix }}=$ $A_{\text {mix }}-E_{\text {mix }}$. No clear preferred ratio has been experimentally established for the two components in the $\mathrm{pDCB} / \mathrm{pDBB}$ solid solution, so a range of selected ratios $\tau$ was considered. The 15 proportion of molecular substitutions along the ribbons and the proportion up the stacks was calculated.

\subsection{Calculations on eniluracil}

The binary, static disorder in eniluracil occurs with respect to the exchange of the $\mathrm{C}_{4}=\mathrm{O}_{8}$ carbonyl group with the $\mathrm{C}_{6}-\mathrm{H}_{6}$ group (see

${ }_{20}$ Fig. 1). An initial, ordered crystal structure was obtained in the form of the $P 2_{1} / c$ structure denoted ak56 from the CSP study of ref $^{5}$, which is the lattice energy minimum corresponding to the major disorder component of the experimental structures. The second component corresponds to rotation of the molecule around 25 the ethynyl bond $\left(\mathrm{C}_{10}-\mathrm{C}_{9}\right)$ axis within the crystal lattice. The crystal structure is symmetric with respect to this rotation being performed on all molecules simultaneously. Three single crystals studied in ref $^{5}$ have proportions of the minor component in the range $0.25-0.3$ within a disorder model that encompasses all 30 sites (a fourth single crystal had a ratio of 0.16 in the same disorder model but was better represented by a different, more complicated disorder model which is not considered in this study). For this reason, this study particularly focuses on the ratio $\tau_{\text {exp }}=0.27$, which is typical of the experimental disorder ratios. ${ }_{35}$ Since the two components have identical lattice energies when unmixed, an ensemble with $\mu=0$ must give a disorder ratio of 0.5 .

The crystal structure of eniluracil has three main features: hydrogen-bonded base-paired ribbons parallel to the $b$ axis, close40 contacted sheets in which these ribbons interdigitate, and stacks of the sheets parallel to the $a$ axis (see Figs. 4,5). The conventional unit cell already contains a ribbon-ribbon interdigitation, so a $2 \times 2 \times 1$ supercell (containing 16 molecules) was chosen to model the disorder in this system, in order to 45 capture orientational variation with respect to all three structural features. A larger supercell would capture more variations within the same features, and is therefore not necessary in this study. Again, symmetry with respect to the two disorder components means that configurations with a disorder ratio of $<0.5(n<8)$ 50 map exactly to those with $\tau>0.5(8<n \leq 16)$. Only those configurations with a ratio $\tau \leq 0.5$ were therefore evaluated. An ensemble of 2565 symmetry-unique supercell configurations were evaluated and included in ensembles. To analyse the structural motifs, the proportions of polar and uniform non-polar 55 ribbons were evaluated, as were the proportion of interdigitating molecules with matching and anti-parallel orientations, and the proportion of orientation changes along stacks of molecules.

\section{Results}

\section{1 p-dichlorobenzene/p-dibromobenzene solid solution}

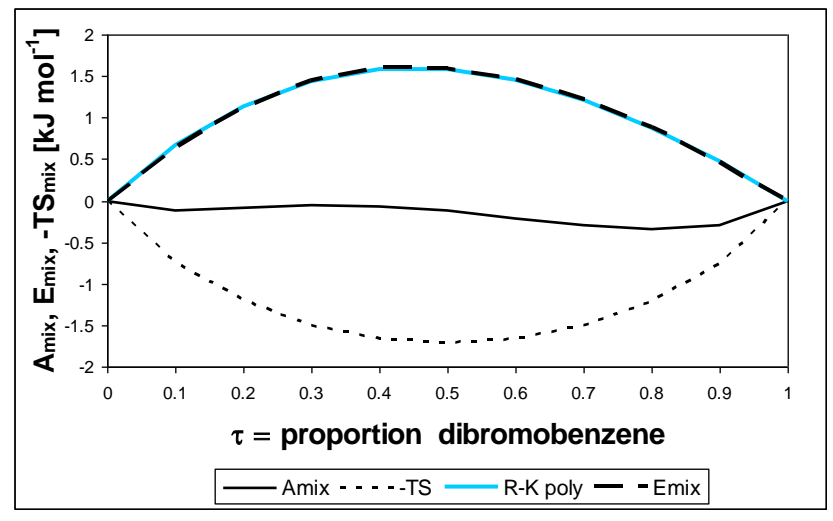

Fig. 6 Lattice free energy of mixing $\left(A_{m i x}\right)$, lattice energy of mixing $\left(E_{m i x}\right)$, and configurational entropic contribution $\left(\mathrm{T} S_{m i x}\right)$ as a function of $\mathrm{pDBB}$ substitution for the $\mathrm{pDCB} / \mathrm{pDBB}$ solid solution. The Redlich-Kister polynomial fitted to the lattice energy of mixing is shown as ' $\mathrm{R}-\mathrm{K}$ poly'.

65 The fit is so close as to make the two lines almost indistinguishable.

The mixing energies and entropies calculated at varying disorder ratios $\tau$ (Fig. 6) show that the configurational entropy contribution to the free energy, TS, is more or less symmetric 70 around a minimum at $\tau \approx 0.5$. However, the lattice energy change of mixing is positive and reaches a maximum at slightly lower than 0.5 . To test whether this system behaves in a similar way to inorganic solid solutions, the lattice energy was fitted to the Redlich-Kister polynomial, ${ }^{21}$

${ }_{75} E_{m i x}=\tau(1-\tau)\left[W_{0}+W_{1}(2 \tau-1)\right]$,

as is typically done in the thermodynamic analysis of mineral solid solutions. ${ }^{67}$ Fig. 6 shows that the behaviour is analagous, as that data fits the equation well with $\mathrm{W}_{0}=6.3 \mathrm{~kJ} \mathrm{~mol}^{-1}$ and $\mathrm{W}_{1}=-$ $1.4 \mathrm{~kJ} \mathrm{~mol}^{-1}$. Hence, the formation of the solid solution is 80 endothermic at all ratios, with the energetic cost of doping DCB with DBB being somewhat higher $\left(\mathrm{W}_{0}-\mathrm{W}_{1}=7.7 \mathrm{~kJ} \mathrm{~mol}^{-1}\right)$ than the cost of doping DBB with DCB $\left(\mathrm{W}_{0}+\mathrm{W}_{1}=5.0 \mathrm{~kJ} \mathrm{~mol}^{-1}\right)$. This asymmetry is expected from the larger radius of $\mathrm{Br}$ compared to $\mathrm{Cl}$, as the incorporation of a larger atom in a smaller atom site 85 should lead to a larger elastic strain in the lattice than vice versa.

The free energy of mixing arising from the combination of entropy and energy has two minima, at $\tau \approx 0.1$, and a slightly deeper one at $\tau \approx 0.8$. Both minima are shallow, less than $0.5 \mathrm{~kJ}$ $\mathrm{mol}^{-1}$. Overall, the free energy of mixing remains negative (i.e. 90 favourable) at all ratios. The ratio obtained with $\mu=0$ is $\tau \approx 0.45$.

The proportion of molecular alternations (one molecule then the other) along both the ribbons and the stacks is within $1 \%$ of the value $2 *(\tau(1-\tau))$ for all motifs and at all ratios. This agreement with the probability for a completely random distribution 95 suggests that the placement of one molecule or the other in each lattice site is genuinely random at all ratios.

\subsection{Eniluracil}

The results in Table 1 contrast the energies of ordered structures with those obtained for various ensemble averages over the 
configurations of the $2 \times 2 \times 1$ supercell. These include both $\tau=0.5$ $\left(\mu=0\right.$ ), and the $\tau_{\exp }=0.27$ experimental average (see Section 2.3). Unexpectedly, both of these averages produce free energies that compare unfavourably with the ordered, single orientation 5 structure. Upon investigation, it was noted that certain classes of configurations with highly unfavourable energies had close oxygen-oxygen contacts (see Fig. 5(c)) within the hydrogen bonded ribbons, which would be unlikely to form during crystal growth (see section 4.1). A typical configuration containing a 10 ribbon with a close $\mathrm{O} \cdots \mathrm{O}$ contact is about $15 \mathrm{~kJ} \mathrm{~mol}^{-1}$ less stable than structures with the polar or non-polar ribbons. However a sufficient number of such configurations are generated by SOD as to make a significant contribution to ensemble averages. That is, even though each of these configurations individually has a 15 low probability of occurring (as dictated by the exponential in eqn 3), the very high proportion of them in the set of all configurations means that close $\mathrm{O} \cdots \mathrm{O}$ contacts would be expected. This results in an unstable average structure, even taking configurational entropy into account. Given the growth 20 mechanistic implausibility of such configurations a new average was calculated, excluding any configuration that did not consist entirely of polar or uniform non-polar ribbons. This contains just 28 configurations, and is referred to as the 'ordered-ribbon' average since any disorder is now the result of changes between 25 ribbons.

Table 1 Eniluracil lattice and free energies

\begin{tabular}{cccc}
\hline $\begin{array}{c}\text { Average } \\
\text { scheme }\end{array}$ & $\begin{array}{c}\text { Lattice energy } \\
{\left[\mathrm{kJ} \mathrm{mol}^{-1}\right]}\end{array}$ & $\begin{array}{c}-\mathrm{TS} \\
{\left[\mathrm{kJ} \mathrm{mol}^{-1}\right]}\end{array}$ & $\begin{array}{c}\text { Free energy } \\
{\left[\mathrm{kJ} \mathrm{mol}^{-1}\right]}\end{array}$ \\
$\begin{array}{c}\text { Ordered } \\
(a k 56)^{\mathrm{a}}\end{array}$ & -117.95 & 0 & -117.95 \\
$\begin{array}{c}\text { Ordered, } \\
\text { polar }\end{array}$ & -116.92 & 0 & -116.92 \\
$\begin{array}{c}\mathrm{O} \cdots \text { close- }^{\mathrm{b}} \\
\text { contact }\end{array}$ & -101.97 & 0 & -101.97 \\
$\tau=0.5(\mu=0)$ & -112.35 & -1.41 & -113.77 \\
$\tau_{\text {exp }}=0.27$ & -113.357 & -1.16 & -114.52 \\
$\tau_{\text {exp }}$ Ordered $_{\text {ribbon }}^{\mathrm{d}}$, & -117.27 & -0.67 & -117.94 \\
\hline
\end{tabular}

a 'Ordered' indicates the basic, ordered crystal structure with no orientational changes ( $a k 56$ ).

b 'Ordered, polar' indicates a structure consisting of polar ribbons which 30 is orientationally ordered.

c ' $\mathrm{O} \cdots \mathrm{O}$ close contact' indicates a structure in which all ribbons contain

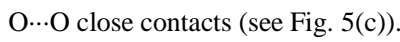

d 'Ordered ribbon' indicates the ensemble of ordered ribbon configurations, as described in section 3.2.

${ }_{35}$ To investigate the hypothetical effects of different disorder ratios, the energies calculated for different $\tau$ values using ordered-ribbon averages are plotted in Fig. 7. As can be seen, the lattice free energy, $A$, has a minimum at $\tau \approx 0.1$, then rises at higher disorder ratios, becoming unfavourable to disorder (i.e. higher than the 40 ordered system) above $\tau \approx 0.3$. By contrast, the average lattice energy decreases the stability, while the entropic contribution increases the stability monotonically with $\tau$.

Table 2 Mechanical and structural properties for eniluracil

\begin{tabular}{cccc}
\hline Property & $\begin{array}{c}\text { Ordered } \\
(a k 56)\end{array}$ & Ordered, polar & $\begin{array}{c}\text { Ordered ribbon, } \\
\tau_{\exp }=0.27\end{array}$ \\
$\rho\left[\mathrm{g} \mathrm{cm}^{-3}\right]$ & 1.529 & 1.524 & 1.525 \\
$C_{11}[\mathrm{GPa}]^{\mathrm{a}}$ & 17.2 & 16.9 & 16.9 \\
$C_{22}[\mathrm{GPa}]$ & 41.3 & 39.7 & 43.6 \\
$C_{33}[\mathrm{GPa}]$ & 31.2 & 29.4 & 30.3 \\
$C_{44}[\mathrm{GPa}]$ & 10.5 & 10.4 & 9.6 \\
$C_{55}[\mathrm{GPa}]$ & 15.2 & 14.6 & 15.1 \\
$C_{66}[\mathrm{GPa}]$ & 4.7 & 4.0 & 4.3 \\
Proportion & 0 & 1 & 0.35 \\
polar ribbons & & & \\
Proportion & 1 & 1 & 0.57 \\
matching & & & \\
interdigitations & & 1 & \\
Proportion & 0 & &
\end{tabular}

${ }_{45}{ }^{a}$ The diagonal elements $\left(\mathrm{C}_{\mathrm{jj}}\right)$ of the elastic constant matrix, describing stiffness to uniaxial compression $(j=1-3)$ and shear $(j=4-6)$. The matrix is calculated in an axis system with $\mathrm{z}$ parallel to the crystal $\mathrm{c}$ axis, $\mathrm{x}$ parallel to the reciprocal lattice $\mathrm{a}^{*}$ axis, and y chosen to give an orthogonal right-handed axis system.

$50{ }^{\mathrm{b}}$ Since the average was only taken with respect to ribbons that were either polar or uniform non-polar, only the proportions of polar ribbons, matching interdigitated molecules and matching stacks are quotedIt is emphasized that the new ensemble was constructed by deliberate exclusion of configurations containing structural features that were judged 55 to be unrealistic. The manual exclusion of certain configurations from ensembles has previously been used, for example, in the modelling of aluminosilicates, ${ }^{68}$ to remove configurations that violate Lowenstein's rule ${ }^{69}$.

The physically more realistic ordered-ribbon average was used to ${ }_{60}$ calculate the elastic constant matrix $\left(C_{i j}\right)$, as a representation of the single crystal mechanical properties (see Table 2). The most significant weakening in the disordered structure relative to the ordered structure is in $\mathrm{C}_{44}$ and $\mathrm{C}_{66}$, representing weakness to shear along the $a$ and $c$ axes; these are parallel to the molecular 65 sheets and the molecular stacks (see Fig. 4), respectively. Examination of the structural motifs (Table 2) shows a preference for uniform non-polar as opposed to polar ribbons, and a slight preference for interdigitating molecules at ribbon junctions to be of the same orientation. The proportion of mismatches in the 70 orientation of the molecules within stacks $(0.36)$ is only slightly lower than the value $((0.27 \times 0.73) \times 2 \approx 0.4)$ that would indicate randomness subject to the enforced disorder ratio, indicating a low degree of ordering in favour of matching orientations within the stacks. The structural motifs therefore suggest a degree of 75 ordering with respect to ribbons and sheets, but only a very slight degree of ordering with respect to stacks. This is consistent with interlayer interactions being the weakest among the three main structural motifs. 


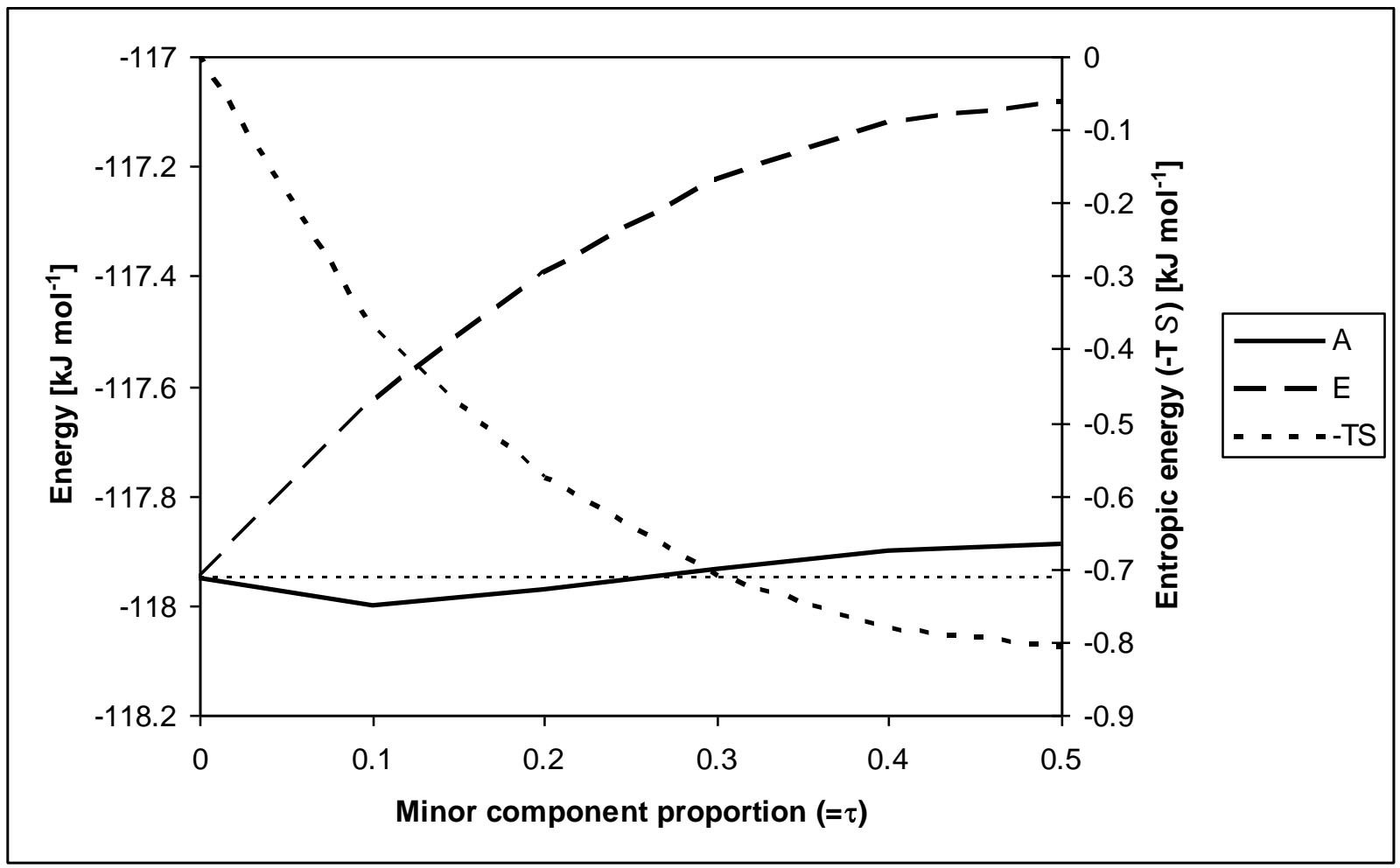

Fig. 7 Lattice free energy $(A)$, average lattice energy $(E)$, and configurational entropic energy (TS) as a function of minor disorder component proportion for eniluracil. $A$ and $E$ follow the scale on the left axis, TS follows the scale on the right. The light broken line represents the lattice energy of the ordered system.

\section{${ }_{5}$ 4. Discussion}

\subsection{Energies and ensemble calculations for disordered molecular crystals}

The static disorder in these, and most molecular crystals, is determined during crystallisation. The high barriers to solid-state

10 rearrangement mean that a disordered crystal will not be in thermal equilibrium with respect to the disorder components. The disorder is determined by the incorporation of the molecules into the growing crystal, which occurs at relatively low temperatures (typically $<373 \mathrm{~K}$ ) and is determined by the

15 kinetics of the attachment of molecules to the structurally distinct crystal faces. This affects two aspects of the combination scheme used to calculate average values in this study. The first of these is the use of the grand canonical ensemble with a chemical potential $\mu$ to calculate averages for selected disorder ratios, $\tau$. The

20 physical significance of $\mu_{\tau}$ therefore includes the composition of the surrounding solution, and the ease of adding one disorder component or another at each type of growth site on each crystal face. Although the composition of the solution or melt is clearly a major factor in determining the disorder ratio in solid solutions, 25 the complexity of the growth mechanism for different faces of organic crystals implies that the physical significance of $\mu_{\tau}$ with regards to a real single crystal with disorder ratio $\tau$ is unclear. It may also include history-dependent factors such as defects and to some extent $\mu$ may also include energetic information that is 30 otherwise missing from our model, such as a correction for the divergence between the supercell model and the real nonperiodic crystal.
The dynamics of crystallisation also justify the restriction of the set of configurations included in the ensemble averages to a 35 selected subset, as in the 'ordered-ribbon' configurations used for eniluracil. To confirm that it is reasonable to assume that the growth mechanisms of the crystal would exclude such contacts, we have calculated the binding energies of eniluracil trimers (see Supporting Information Section S2), the basic units through 40 which the ribbons (Fig. 5) can grow from solution. A trimer with an $\mathrm{O} \cdots \mathrm{O}$ close contact has a binding energy of only $-24.7 \mathrm{~kJ} \mathrm{~mol}^{-}$ ${ }^{1}$, whereas the trimers in the polar and uniform non-polar ribbons have binding energies of $-103.5 \mathrm{~kJ} \mathrm{~mol}^{-1}$ and $-108.3 \mathrm{~kJ} \mathrm{~mol}^{-1}$ respectively. Thus the energy penalty for attaching a molecule 45 with a close $\mathrm{O} \cdots \mathrm{O}$ contact to a ribbon during crystal growth will be even higher than implied by relative energy of the crystalline configurations with the $\mathrm{O} \cdots \mathrm{O}$ close contacts (Table 1). It is possible that a ribbon could switch between polar and uniform non-polar character, but only through vacancy defects. This is not 50 likely to be a major effect, and was not considered further.

Although the exclusion of $\mathrm{O} \cdots \mathrm{O}$ close contact configurations means that the ensemble averages calculated do not represent a full thermodynamic equilibrium with respect to the supercells, it provides a more realistic model for the configurations that can 55 occur in eniluracil crystals. The ability to identify and remove such subsets is a good reason for using symmetry-adapted ensembles to model disordered organic molecular systems in preference to MCX. It seems safer to use a method which identifies all configurations for a given supercell, and then 60 consider whether any high energy configurations that affect the thermodynamic averaging because of their frequency are plausible considering growth mode and available experimental 
data.

\section{2 p-dichlorobenzene/p-dibromobenzene solid solution}

Mixing energies calculated at different compositions (disorder 5 ratios) for the $\mathrm{pDCB} / \mathrm{pDBB}$ solid solution (Fig. 6) show that mixing is always stable with respect to the pure phases (although not by a wide margin, $<0.5 \mathrm{~kJ} \mathrm{~mol}^{-1}$ ), indicating that preparation may be possible at a range of compositions. This is supported by experimental reports. ${ }^{55}$ The shallow minima at $\tau \approx 0.1$ and 0.8 10 indicate that formation will be particularly favourable at these ratios, consistent with one experimental report ${ }^{54}$ that the solid solution exhibits a miscibility gap between $\tau=0.3$ and $\tau=0.72$, However, the existence of this miscibility gap has been questioned following alternative experiments. ${ }^{55}$ The shallowness 15 of the two minima would indicate that the driving force for separation into $\mathrm{Cl}$-rich and $\mathrm{Br}$-rich phases is small.

At all ratios, the disordered system is endothermic with respect to the single component crystals, but the mixing is made stable by consideration of the configurational entropy. This reaches a 20 maximum at $\tau=0.5$ with a value of $-1.72 \mathrm{~kJ} \mathrm{~mol}^{-1}$, matching the binary maximum of $R T \ln 2$. The randomness of the arrangement of the molecules within the main structural motifs (Section 3.1) is consistent with the high degree of configurational entropy. Thus our qualitative results are consistent those obtained for a simpler 25 model $^{13}$ of a 50:50 disordered system which assumed that the configurational entropy was $R T \ln 2$.

Hence, these calculations have revealed that the DCB/DBB solid solution has a shallow miscibility gap, and also that the distribution of the different species is (close to) fully random, 30 neither of which were obvious from casual consideration of the system.

\subsection{Eniluracil}

The most important point to arise from this study of eniluracil is the necessity of excluding from the ensemble used to model the 35 system any configurations containing highly unstable structural features. This is physically reasonable: the repulsion between oxygen atoms makes it highly unlikely that hydrogen bonds would form between molecules to give the ribbon shown in Fig. $5 c$ during crystal growth.

40 When the disorder of eniluracil is treated as an ensemble of 'ordered ribbon' states (those containing only polar or uniform non-polar ribbons, see Section 3.2), the free energy at any disorder ratio is roughly equal to that of the ordered system (Table 1, Fig. 7). Variations in free energy with disorder ratio are 45 very small, of the order of $0.01 \mathrm{~kJ} \mathrm{~mol}^{-1}$. This type of disorder has therefore been shown to be thermodynamically accessible, though not markedly favourable with respect to an ordered system. In addition, the small variations in free energy are consistent with the appearance of different disorder ratios in separate single 50 crystals. ${ }^{5}$ Nonetheless it is evident that there is a minimum in free energy around $\tau \approx 0.1$, and that disorder moves from being stable to being unstable with respect to the ordered system at around $\tau \approx$ 0.3 . Three out of four of the single crystals reported in $^{5}$ have disorder ratios close to this boundary (between $\tau \approx 0.25$ and $\tau \approx$ 550.3 ), while the fourth has a ratio of 0.18 in this disorder model, which is also in the favoured region. The variation in disorder ratio between crystals grown in the same conditions is consistent with the shallowness of the energy minimum.

Many of the energy differences reported in this study are rather 60 small - often less than $1 \mathrm{~kJ} \mathrm{~mol}^{-1}$, as generally expected for energies of mixing. ${ }^{21}$ It is worth noting that considerable cancellation of errors can be expected in relative energies, of different configurations of one supercell. The energy differences discussed here, while small, are therefore significant.

${ }_{65}$ Hence, these calculations have demonstrated what could only be qualitatively inferred from the crystal energy landscapes of ref, ${ }^{5}$ that the disorder in eniluracil is effectively disorder between polar and uniform non-polar hydrogen bonded ribbons. It has also been shown that this disorder results in a mechnically weaker crystal. 70 The ability to computationally model disorder once its presence has been detected by X-ray crystallography is therefore of potential relevance to processing and handling of drug molecules.

\subsection{Further work}

This study has introduced the capability to computationally 75 model static disordered organic systems that are found experimentally. This will help to assess the validity of and interpretation of crystallographic disorder models. It is hoped that the approach will be further refined by comparison with the more detailed information on disordered systems becoming available 80 through the study of diffuse scattering. ${ }^{9}$ The scheme presented here allows the evaluation of stability for a disordered system, once a structure and two disorder components have been identified. Progress towards genuinely predicting a tendency to crystallize with disorder will require methods of analysing the 85 crystal energy landscapes of energetically favourable ordered structures to determine possible disorder components. For example, the eniluracil crystal energy landscape had various low energy structures which would be virtually identical if $\mathrm{C}_{6}-\mathrm{H}_{6}$ and $\mathrm{C}_{4}=\mathrm{O}_{8}$ (Fig. 1) were not distinct. Prediction of a potential solid 90 solution would require identification of isomorphic structures on the crystal energy landscapes of the two molecules.

\section{Conclusions}

A method has been introduced for computational modelling of organic molecular crystals with binary, static disorder. The basis 95 of this method is the implementation of a symmetry-adapted ensemble model through a combination of the SOD ${ }^{18}$ program for generating symmetry relationships and the DMACRYS ${ }^{53}$ program to calculate lattice energies and properties. Energies have been calculated for the complete range of disorder ratios 100 using the grand canonical ensemble. The new method has been used to model the solid solution of $\alpha$ - $p$-dichlorobenzene and $p$ dibromobenzene and the orientational disorder of 5-ethynyl uracil (eniluracil). In both cases, it was shown that disorder was endothermic, and only became favourable when the 105 configurational entropy was also considered. The variation in free energy across a range of hypothetical disorder ratios was calculated for both systems, giving small variations and therefore suggesting a variable ratio in both cases. This is consistent with experimental observation. Evaluation of mechanical properties 110 for the eniluracil system shows that the presence of the disorder has a macroscopic effect, mechanically weakening the crystal relative to the analogous ordered structure. Examination of structural motifs and of the calculated entropies for the two 
systems suggests that eniluracil has significant ordering in the arrangement of the differently oriented molecules, but the solid solution of $\mathrm{pDCB}$ and $\mathrm{pDBB}$ is random in its distribution of different molecules.

5 The scheme presented in this study for evaluating the ensemble averages allows for the manual removal of configurations which are unphysical in that they are unlikely to occur during crystal growth and yet affect ensemble averages because of their number. Greater understanding of the mechanisms of growth, and the 10 kinetic barriers to solid state thermodynamic equilibration of organic crystals will allow the development of the study of disorder and solid solution and impurity incorporation in organic crystals.

15 ACKNOWLEDGMENTS Prof. Bouke van Eijck for useful discussions. The Basic Technology program of the Research Councils UK for funding "Control and Prediction of the Organic Solid State: Translating the Technology" www.cposs.org.uk (EP/F03573X/1).

20

\section{Notes and references}

${ }^{a}$ Department of Chemistry, University College London, 20 Gordon Street, London, WC1H 0AJ, United Kingdom. Fax: +44(0)20 7679 7463; Tel: +44 (0)20 7679 4665; E-mail: m.habgood@ucl.ac.uk

$25 \uparrow$ Electronic Supplementary Information (ESI) available: derivation of the chemical potential, $\mu_{\tau}$, trimer energy calculations for eniluracil. See DOI: $10.1039 / \mathrm{b} 000000 \mathrm{x} /$

\section{Reference List}

1. F. H. Allen, Acta Crystallogr. , Sect. B, 2002, 58, 380-388.

2. C. C. Wilson, Crystallography Reviews, 2009, 15, 3-56.

3. P. Muller, Crystallography Reviews, 2009, 15, 57-83.

4. D. Watkin, J. Appl. Crystallogr., 2008, 41, 491-522.

5. R. C. B. Copley, S. A. Barnett, P. G. Karamertzanis, K. D. M. Harris, B. M. Kariuki, M. C. Xu, E. A. Nickels, R. W. Lancaster and S. L. Price, Cryst. Growth Des., 2008, 8, 3474-3481.

6. L. H. Thomas, J. M. Cole and C. C. Wilson, Acta Crystallogr., Sect. C, 2008, 64, O296-O302.

7. L. H. Thomas, T. R. Welberry, D. J. Goossens, A. P. Heerdegen, M. J. Gutmann, S. J. Teat, P. L. Lee, C. C. Wilson and J. M. Cole, Acta Crystallogr. , Sect. B, 2007, 63, 663-673.

8. E. J. Chan, T. R. Welberry, D. J. Goossens and A. P. Heerdegen, J. Appl. Crystallogr., 2010, 43, 913-915.

45

9. T. R. Welberry, Diffuse X-ray Scattering and Models of Disorder, Oxford University Press, 2004.

10. T. Wagner and A. Schonleber, Acta Crystallogr., Sect. B, 2009, 65, 249-268.

11. S. L. Price, Int. Rev. Phys. Chem., 2008, 27, 541-568.

13. B. P. van Eijck, Phys. Chem. Chem. Phys., 2002, 4, 4789-4794.

14. N. Issa, P. G. Karamertzanis, G. W. A. Welch and S. L. Price, Cryst. Growth Des., 2009, 9, 442-453.

15. L. Carlucci and A. Gavezzotti, Chem. Eur. J, 2005, 11, 271-279.

16. S. A. Barnett, A. T. Hulme, N. Issa, T. C. Lewis, L. S. Price, D. A. Tocher and S. L. Price, New J. Chem., 2008, 32, 1761-1775.

17. A. Torrisi, C. K. Leech, K. Shankland, W. I. F. David, R. M. Ibberson, J. Benet-Buchholz, R. Boese, M. Leslie, C. R. A. Catlow and S. L. Price, J. Phys. Chem. B, 2008, 112, 3746-3758.

18. R. Grau-Crespo, S. Hamad, C. R. A. Catlow and N. H. de Leeuw, Journal of Physics: Condensed Matter, 2007, 19, Art-256201.

19. S. Benny, R. Grau-Crespo and N. H. de Leeuw, Phys. Chem. Chem. Phys., 2009, 11, 808-815.

20. R. Grau-Crespo, A. Y. Al-Baitai, I. Saadoune and N. H. de Leeuw, J. Phys,-Condens. Mat., 2010, 22, Art-255401.

21. S. E. Ruiz-Hernandez, R. Grau-Crespo, A. R. Ruiz-Salvador and N. H. de Leeuw, Geochimica et Cosmochimica Acta, 2010, 74, 1320-1328.

22. J. Graciani, S. Hamad and J. F. Sanz, Phys. Rev. B, 2009, 80, Art184112 .

23. Q. Wang and N. H. de Leeuw, Mineral. Mag., 2008, 7, 525-529.

24. A. Meyer, Ph. D'Arco, R. Orlando and R. Dovesi, J. Phys. Chem. $C, 2009,113,14507-14511$.

25. C. L. Freeman, N. L. Allan and W. van Westrenen, Phys. Rev. B, 2006, 74, Art-134203.

26. I. T. Todorov, N. L. Allan, M. Y. Lavrentiev, C. L. Freeman, C. E. Mohn and J. A. Purton, J. Phys,-Condens. Mat., 2004, 16, S2751-S2770.

27. J. A. Purton, N. L. Allan, M. Y. Lavrentiev, I. T. Todorov and C. L. Freeman, Chemical Geology, 2006, 225, 176-188.

28. V. L. Vinograd, J. D. Gale and B. Winkler, Phys. Chem. Miner., 2007, 34, 713-725.

29. V. L. Vinograd, B. P. Burton, J. D. Gale, N. L. Allan and B. Winkler, Geochimica et Cosmochimica Acta, 2007, 71, 974-983. 
30. M. Y. Lavrentiev, R. Drautz, D. Nguyen-Manh, T. P. C. Klaver and S. L. Dudarev, Phys. Rev. B, 2007, 75, Art-014208.

31. M. Y. Lavrentiev, S. L. Dudarev and D. Nguyen-Manh, Journal of Nuclear Materials, 2009, 386, 22-25.

$5 \quad$ 32. V. L. Vinograd and B. Winkler, in Theoretical and Computational Methods in Mineral Physics, 2010 pp 413-436.

33. M. J. Akhtar, C. R. A. Catlow, B. Slater, A. M. Walker and S. M. Woodley, Chem. Mater., 2006, 18, 1552-1560.

34. G. W. Watson and D. J. Willock, Chem. Commun., 2001, 10761077.

35. A. Bosenick, M. T. Dove, V. Heine and C. A. Geiger, Phys. Chem. Miner., 2001, 28, 177-187.

36. A. Zunger, S. H. Wei, L. G. Ferreira and J. E. Bernard, Phys. Rev. Lett., 1990, 65, 353-356.

37. N. A. Murugan, J. Phys. Chem. B, 2005, 109, 23955-23962.

38. N. A. Murugan and A. Sayeed, J. Chem. Phys., 2009, 130, 204514 (Art. No.).

39. B. Verberck, G. A. Vliegenthart and G. Gompper, J. Chem. Phys., 2009, 130, Art-154510.

40. F. G. Vogt, J. A. Vena, M. Chavda, J. S. Clawson, M. Strohmeier and M. E. Barnett, J. Mol. Struct., 2009, 932, 16-30.

41. D. Britton, Acta Crystallogr., Sect. B, 2009, 65, 54-58.

42. P. M. Bhatt and G. R. Desiraju, Chem. Commun., 2007, 20572059.

43. A. J. Florence, C. K. Leech, N. Shankland, K. Shankland and A. Johnston, CrystEngComm, 2006, 8, 746-747.

44. M. Polito, E. D'Oria, L. Maini, P. G. Karamertzanis, F. Grepioni, D. Braga and S. L. Price, CrystEngComm, 2008, 10, 1848-1854.

45. T. R. Welberry, Acta Cryst. A, 2000, 56, 348-358.

46. T. Yildirim, P. M. Gehring, D. A. Neumann, P. E. Eaton and T. Emirck, Carbon, 1998, 36, 809-815.

47. J. P. Amoureux, M. Bee and J. C. Damien, Acta Crystallogr., Sect. B, 1980, 36, 2633-2636.

48. P. Launois, S. Ravy and R. Moret, Phys. Rev. B, 1995, 52, 54145425.
49. M. Tremayne, L. Grice, J. C. Pyatt, C. C. Seaton, B. M. Kariuki, H. H. Y. Tsui, S. L. Price and J. C. Cherryman, J. Am. Chem. Soc., 2004, 126, 7071-7081.

50. P. Derollez, N. T. Correia, F. Danede, F. Capet, F. Affouard, J. Lefebvre and M. Descamps, Acta Crystallogr. , Sect. B, 2005, 61, 329-334.

51. C. W. Lehmann and F. Stowasser, Chem. Eur. J, 2007, 13, 29082911.

52. G. D. Enright, V. V. Terskikh, D. H. Brouwer and J. A. Ripmeester, Cryst. Growth Des., 2007, 7, 1406-1410.

53. S. L. Price, M. Leslie, G. W. A. Welch, M. Habgood, L. S. Price, P. G. Karamertzanis and G. M. Day, Phys. Chem. Chem. Phys., 2010, 12, 8478-8490.

54. M. T. Calvet, M. A. Cuevas-Diarte, Y. Haget, P. R. van der Linde and H. A. J. Oonk, CALPHAD, 1991, 15, 225-234.

55. A. P. Bussandri, M. J. Zuriaga and C. A. Martin, J. Phys. Chem. Solids, 1998, 59, 201-209.

56. A. J. Stone, J. Chem. Theory Comput., 2005, 1, 1128-1132.

57. M. J. Frisch, G. W. Trucks, H. B. Schlegel, G. E. Scuseria, M. A. 55 Robb, J. R. Cheeseman, J. Montgomery, T. Vreven, K. N. Kudin, J. C. Burant, J. M. Millam, S. S. Iyengar, J. Tomasi, V. Barone, B. Mennucci, M. Cossi, G. Scalmani, N. Rega, G. A. Petersson, H. Nakatsuji, M. Hada, M. Ehara, K. Toyota, R. Fukuda, J. Hasegawa, M. Ishida, T. Nakajima, Y. Honda, O. Kitao, H. Nakai, M. Klene, X. Li, J. E. Knox, H. P. Hratchian, J. B. Cross, V. Bakken, C. Adamo, J. Jaramillo, R. Gomperts, R. E. Stratmann, O. Yazyev, A. J. Austin, R. Cammi, C. Pomelli, J. Ochterski, P. Y. Ayala, K. Morokuma, G. A. Voth, P. Salvador, J. J. Dannenberg, V. G. Zakrzewski, S. Dapprich, A. D. Daniels, M. C. Strain, O. Farkas, D. K. Malick, A. D. Rabuck, K. Raghavachari, J. B. Foresman, J. V. Ortiz, Q. Cui, A. G. Baboul, S. Clifford, J. Cioslowski, B. B. Stefanov, G. Liu, A. Liashenko, P. Piskorz, I. Komaromi, R. L. Martin, D. J. Fox, T. Keith, M. A. Al Laham, C. Y. Peng, A. Nanayakkara, M. Challacombe, P. M. W. Gill, B. Johnson, W. Chen, M. W. Wong, C. Gonzalez and J. A. Pople. Gaussian 03 Gaussian Inc. Wallingford CT. (2004).

58. D. S. Coombes, S. L. Price, D. J. Willock and M. Leslie, J. Phys. Chem., 1996, 100, 7352-7360.

59. D. E. Williams and S. R. Cox, Acta Crystallogr., Sect. B, 1984, 40, 404-417.

60. S. R. Cox, L. Y. Hsu and D. E. Williams, Acta Crystallogr., Sect A., 1981, 37, 293-301.

61. L. Y. Hsu and D. E. Williams, Acta Crystallogr., Sect A., 1980, 36, 277-281. 
62. W. D. S. Motherwell, H. L. Ammon, J. D. Dunitz, A.

Dzyabchenko, P. Erk, A. Gavezzotti, D. W. M. Hofmann, F. J. J. Leusen, J. P. M. Lommerse, W. T. M. Mooij, S. L. Price, H. Scheraga, B. Schweizer, M. U. Schmidt, B. P. van Eijck, P. Verwer and D. E. Williams, Acta Crystallogr., Sect. B, 2002, 58, 647-661.

63. G. M. Day, S. L. Price and M. Leslie, Cryst. Growth Des., 2001, 1, 13-27.

64. R. Grau-Crespo, K. C. Smith, T. S. Fisher, N. H. de Leeuw and U. V. Waghmare, Phys. Rev. B, 2009, 80, 174117.

65. A. J. Pertsin and A. I. Kitaigorodsky, The Atom-Atom Potential Method. Applications to Organic Molecular Solids, SpringerVerlag, Berlin, 1987.

66. A. Belaaraj. PhD thesis. 1992. Universidad de Barcelona, Spain.

67. M. Prieto, Reviews in Mineralogy and Geochemistry, 2009, 70, 47-85.

68. A. R. Ruiz-Salvador, N. Almora-Barrios, A. Gomez and D. W. Lewis, Phys. Chem. Chem. Phys., 2007, 9, 521-532.

69. W. Lowenstein, American Minerologist, 1954, 39, 92. 